\title{
Viscoelectroelastic behavior of heterogeneous piezoelectric solids
}

Jiangyu Li, Martin L. Dunn

Jiangyu Li, Martin L. Dunn, "Viscoelectroelastic behavior of heterogeneous piezoelectric solids," Proc. SPIE 3992, Smart Structures and Materials 2000: Active Materials: Behavior and Mechanics, (14 June 2000); doi: 10.1117/12.388217

Event: SPIE's 7th Annual International Symposium on Smart Structures and Materials, 2000, Newport Beach, CA, United States 


\title{
Viscoelectroelastic behavior of heterogeneous piezoelectric solids
}

\author{
Jiang Yu Li and Martin L. Dunn ${ }^{\mathrm{b}}$ \\ ${ }^{a}$ Division of Engineering and Applied Science, California Institute of Technology \\ ${ }^{b}$ Department of Mechanical Engineering, University of Colorado at Boulder
}

\begin{abstract}
We study the viscoelectroelastic behavior of heterogeneous piezoelectric solids, focusing on the connection between heterogeneity and coupled mechanical and electrical relaxations. Our approach is based on the existence of a correspondence between quasistatic viscoelectroelasticity and static piezoelectricity when linear constitutive response exists. We couple this correspondence principle with micromechanics models to predict the overall behavior of heterogeneous piezoelectric solids in terms of microstructural details. We devote specific attention to a class of two-phase materials consisting of a lossless piezoelectric phase embedded in a lossy matrix and obtain closed form expressions for the effective complex electroelastic moduli. Numerical results are presented and discussed, and reasonable agreement with experiment is observed.
\end{abstract}

Keywords: piezoelectricity, viscoelasticity, composites, micromechanics

\section{INTRODUCTION}

Widespread practical applications of piezoelectric solids in numerous technological fields have fueled the understanding of many of their physical properties. A set of properties that has received little study, though, are the complex elastic, dielectric, and piezoelectric constants. Actually, the first two have received considerable attention in uncoupled elastic and dielectric solids and excellent texts exist ${ }^{1-3}$. Of course the complex elastic and dielectric constants are associated with mechanical and electrical losses in the material, respectively, and in linear theoretical treatments, alternative but equivalent representations exist to the complex moduli approach.

Complex piezoelectric constants, however, have received far less attention in the literature, but they present many interesting features. Their complex parts do not necessarily represent losses, and they are influenced by both the mechanical and electric losses and their coupling through the piezoelectric effect. The rigorous thermodynamic treatment of complex piezoelectric constants has existed for quite some time ${ }^{4-7}$ and a handful of observations exist. In most of the observed cases, the source of the complex piezoelectric constants was heterogeneity at some length scale. These include ferroelectric single crystals with domain heterogeneities, ${ }^{8}$ polycrystalline ceramics, ${ }^{9-11}$ piezoelectric polymers with preferentially-oriented crystalline phases, ${ }^{12}$ bone, ${ }^{13}$ and composites consisting of piezoelectric ceramics in a polymer matrix. ${ }^{14-17}$ The role of complex piezoelectric constants in polymer matrix composites is especially significant because of the many transducer applications of such materials. In underwater sonar applications, temperature changes can significantly affect mechanical and electric losses in the polymer phase, which in turn impact the complex piezoelectric constants in a nontrivial way.

Although experimental studies of complex piezoelectric constants in heterogeneous media are limited, corresponding modeling efforts are rarer, even though substantial effort has been directed toward micromechanical modeling of elastic, dielectric, and piezoelectric constants of heterogeneous solids in the absence of losses. ${ }^{18-36}$ In this work we study the effect of relaxation in the phases of a heterogeneous solid on the overall response of the solid. Our approach follows along the lines of similar efforts in uncoupled viscoelastic composites. ${ }^{37}-40$ Our focus here is not on the detailed mechanisms of relaxation in individual phases, but instead on the interaction of these phase relaxations and their influence on the overall behavior of the heterogeneous solid.

\section{BASIC EQUATIONS}

Uncoupled elastic and dielectric relaxations of materials have been studied extensively and integral constitutive equations have been used to express the dependence of the current material response on the mechanical and electric loading history. In a similar manner, the constitutive equations for the linear viscoelectroelastic effect, where the elastic and dielectric relaxation are coupled through the piezoelectric effect can be written as: 


$$
\begin{aligned}
& \sigma_{i j}(\mathbf{x}, t)=\int_{-\infty}^{t}\left[C_{i j k l}(\mathbf{x}, t-\tau) \frac{d \varepsilon_{k l}(\mathbf{x}, \tau)}{d \tau}-e_{i j k}(\mathbf{x}, t-\tau) \frac{d E_{k}(\mathbf{x}, \tau)}{d \tau}\right] d \tau \\
& D_{i}(\mathbf{x}, t)=\int_{-\infty}^{t}\left[e_{i k l}(\mathbf{x}, t-\tau) \frac{d \varepsilon_{k l}(\mathbf{x}, \tau)}{d \tau}+\kappa_{i k}(\mathbf{x}, t-\tau) \frac{d E_{k}(\mathbf{x}, \tau)}{d \tau}\right] d \tau
\end{aligned}
$$

where $\sigma_{i j}$ and $\varepsilon_{i j}$ are stress and strain tensors, respectively; $D_{i}$ and $E_{k}$ are the electric displacement and field, respectively; $C_{i j k l}$, $e_{i j k}$, and $\kappa_{i k}$ are elastic, piezoelectric, and dielectric moduli, respectively; $\mathbf{x}$ is the position vector of a material point; $t$ and $\tau$ are the current and a reference time, respectively. It is observed from the constitutive equations that, i) elastic and electric relaxations are fully coupled through the piezoelectric moduli $e_{i j k}$; ii) by ignoring the piezoelectric relaxation, the familiar constitutive equations for uncoupled mechanical and dielectric relaxations are recovered; iii) the stress and electric displacement depend on the strain and electric field history.

In the analysis that follows, it is convenient to treat the elastic and electric fields on equal footing. To this end, the notation introduced by Barnett and Lothe ${ }^{41}$ is utilized. With this notation, the field variables take the following forms:

$$
\Sigma_{i J}(\mathbf{x}, t)=\left\{\begin{array}{l}
\sigma_{i j}(\mathbf{x}, t) \\
D_{i}(\mathbf{x}, t)
\end{array}, Z_{J i}(t)=\left\{\begin{array}{c}
\varepsilon_{i j}(\mathbf{x}, t) \\
-E_{i}(\mathbf{x}, t)
\end{array}, U_{J}(t)=\left\{\begin{array}{cc}
u_{j}(\mathbf{x}, t) & J=1,2,3 \\
\phi(\mathbf{x}, t) & J=4
\end{array},\right.\right.\right.
$$

and the electroelastic moduli can be written as

$$
E_{i j k l}(\mathbf{x}, t-\tau)=C_{i j k l}(\mathbf{x}, t-\tau), E_{i j 4 l}(\mathbf{x}, t-\tau)=e_{i j l}(\mathbf{x}, t-\tau), E_{i 4 k l}(\mathbf{x}, t-\tau)=e_{i k l}(\mathbf{x}, t-\tau), E_{i 44 l}(\mathbf{x}, t-\tau)=-\kappa_{i l}(\mathbf{x}, t-\tau) .
$$

With the electroelastic variables written in this abbreviated notation, the constitutive equations (1) can be rewritten as:

$$
\Sigma_{i J}(\mathbf{x}, t)=\int_{-\infty}^{t} E_{i J K l}(\mathbf{x}, t-\tau) \frac{d Z_{K l}(\mathbf{x}, \tau)}{d \tau} d \tau
$$

The equilibrium and gradient equations for a viscoelectroelastic solid with a distribution of body force and electric charge are:

$$
\begin{aligned}
& \Sigma_{i J, i}(\mathbf{x}, t)+\rho_{J}(\mathbf{x}, t)=0 \\
& Z_{J i}(\mathbf{x}, t)=U_{J, i}(\mathbf{x}, t),
\end{aligned}
$$

where $\rho_{J}$ is the body force when $J$ equals to 1 to 3 , and electric charge when $J$ equals 4 . The problem now is to determine the electroelastic fields modeled by eqs. (4-6), and subjected to the following boundary conditions:

$$
\Sigma_{i J}(\mathbf{x}, t)=\Sigma_{i J}^{0}(t) \text { on } S_{1} \quad \text { and } \quad U_{J}(\mathbf{x}, t)=U_{J}^{0}(\mathbf{x}, t) \text { on } S_{2},
$$

where $S_{1}$ and $S_{2}$ combined to form the surface of the solid. Note that now the field variables are all functions of time. Assume that the viscoelectroelastic solid is subjected to a sinusoidal strain and electric field:

$$
Z_{J i}(\mathbf{x}, t)=Z_{J i}(\mathbf{x}) e^{i \omega t},
$$

where $\omega$ is the circular frequency and $i=\sqrt{-1}$. Substituting eq. (8) into eq. (4), and making the variable change $p=t-\tau$ yields:

$$
\Sigma_{i J}(\mathbf{x}, t)=i \omega Z_{K l}(\mathbf{x}) e^{i \omega t} \int_{0}^{\infty} E_{i J K l}(\mathbf{x}, p) e^{-i \omega p} d p
$$

Let 


$$
E_{i J K l}^{c}(\mathbf{x}, i \omega)=i \omega \int_{0}^{\infty} E_{i J K l}(\mathbf{x}, p) e^{-i \omega p} d p
$$

where the superscript $c$ is used to explicitly denote complex quantities. Equation (9) can be recast as:

$$
\Sigma_{i J}(\mathbf{x}, t)=E_{i J K L}^{c}(\mathbf{x}, i \omega) Z_{K l}(\mathbf{x}, t) \text {, }
$$

which formally resembles the static piezoelectric constitutive equations. $E_{i J K L}^{c}$ can be separated into real and imaginary parts:

$$
E_{i J K L}^{c}(\mathbf{x}, i \omega)=E_{i J K l}^{r}(\mathbf{x}, i \omega)+i E_{i J K l}^{i}(\mathbf{x}, i \omega),
$$

where the superscripts $r$ and $i$ denote the real and imaginary parts of the complex moduli and are called the storage and loss moduli. The ratio of loss moduli to storage moduli is called the loss tangent. Note that we use this terminology even though the complex part of a piezoelectric constant may not represent a loss.

\section{EFFECTIVE COMPLEX ELECTORLEASTIC MODULI OF HETEROGENEOUS SOLIDS}

Consider a multi-phase composite with piecewise homogeneity. When the length scale of heterogeneity is much less than the wavelength of the applied field, the effective complex electroelastic moduli $E_{i J K L}^{*}(i \omega)$ is defined by:

$$
<\Sigma_{i J}(\mathbf{x}, t)>=E_{i J K L}^{*}(i \omega)<Z_{K l}(\mathbf{x}, t)>\text {. }
$$

A correspondence principle can be established between the effective complex electroelastic moduli of heterogeneous viscoelectroelastic solids and the effective static electroelastic moduli of piezoelectric solids. ${ }^{42}$ That is, the effective complex electroelastic moduli of a heterogeneous material are found by replacing the constituent static electroelastic moduli by the constituent complex electroelastic moduli in the relationships between the effective static electroelastic moduli and the constituent static electroelastic moduli of the heterogeneous piezoelectric solid. This correspondence principle can be coupled with any exact results or with a micromechanics model to determine the effective complex moduli of heterogeneous viscoelectroelastic solids.

For the remainder of this section we focus on two-phase composites consisting of one phase that is piezoelectric and lossless (for example, a piezoelectric ceramic) and another phase that is not piezoelectric, but is both elastically and electrically lossy (for example, a polymer). The results obtained here result as special cases of the more general analyses that we have presented elsewhere. ${ }^{42}$ Here we focus primarily on the behavior of the complex piezoelectric constants. More extensive results focused on the complex elastic and dielectric constants are presented elsewhere. ${ }^{42}$

To demonstrate the basic ideas and phenomenology, we first study two one-dimensional composite systems consisting of the two phases described above. In one case the two phases are arranged in parallel and in the other they are arranged in series. The constitutive equations of the two phases and of the composite in both cases are:

$$
\begin{aligned}
& \sigma=C^{c} \varepsilon-e^{c} E \\
& D=e^{c} \varepsilon+\kappa^{c} E
\end{aligned}
$$

We take phase 1 to be the uncoupled (nonpiezoelectric) lossy solid. It has a volume fraction $c_{1}$, elastic constant $C_{1}^{c}=C_{1}^{r}+i C_{1}^{i}$, and dielectric constant $\kappa_{1}^{c}=\kappa_{1}^{r}+i \kappa_{1}^{i}$. Phase 2 is the lossless piezoelectric solid. It has a volume fraction $c_{2}$, elastic constant $C_{2}$, piezoelectric constant $e_{2}$, and dielectric constant $\kappa_{2}$. All three of its constants are real.

When the two phases are arranged in parallel the effective moduli of the composite are given exactly by the linear rule of mixtures. This case is not particularly interesting as the composite always exhibits a real piezoelectric constant, and so we do not discuss it further. More interesting is the case when the two phases are arranged in series. Then the stress and electric displacement in both phases are equal to the average stress and average electric displacement in the composite. The effective moduli of the composite can be obtained as: 


$$
\begin{aligned}
& C^{r}=\frac{C_{1}^{r}\left(c_{1} e_{2}^{2}+c_{1} C_{2} \kappa_{2}+c_{2} C_{2} \kappa_{2}^{r}\right)}{c_{1}^{2} e_{2}^{2}+\left(c_{1} C_{2}+c_{2} C_{1}^{r}\right)\left(c_{1} \kappa_{2}+c_{2} \kappa_{1}^{r}\right)} \\
& C^{i}=\frac{c_{1}\left[\left(c_{1} e_{2}^{2}+c_{2} C_{2} \kappa_{1}^{r}+c_{1} C_{2} \kappa_{2}\right)^{2} C_{1}^{i}-c_{2}^{2} C_{1}^{r 2} e_{2}^{2} \kappa_{1}^{i}\right]}{\left[c_{1}^{2} e_{2}^{2}+\left(c_{1} C_{2}+c_{2} C_{1}^{r}\right)\left(c_{1} \kappa_{2}+c_{2} \kappa_{1}^{r}\right)\right]^{2}} \\
& e^{r}=\frac{c_{2} C_{1}^{r} \kappa_{1}^{r} e_{2}}{c_{1}^{2} e_{2}^{2}+\left(c_{1} C_{2}+c_{2} C_{1}^{r}\right)\left(c_{1} \kappa_{2}+c_{2} \kappa_{1}^{r}\right)} \\
& e^{i}=\frac{c_{1} c_{2} e_{2}\left[c_{2} C_{1}^{r 2} \kappa_{2} \kappa_{1}^{i}+c_{1} C_{1}^{r}\left(e_{2}^{2}+C_{2} \kappa_{2}\right) \kappa_{1}^{i}+\kappa_{1}^{r}\left(c_{1} e_{2}^{2}+c_{2} C_{2} \kappa_{1}^{r}+c_{1} C_{2} \kappa_{2}\right) C_{1}^{i}\right]}{\left[c_{1}^{2} e_{2}^{2}+\left(c_{1} C_{2}+c_{2} C_{1}^{r}\right)\left(c_{1} \kappa_{2}+c_{2} \kappa_{1}^{r}\right)\right]^{2}} \\
& \kappa^{r}=\frac{\kappa_{1}^{r}\left(c_{1} e_{2}^{2}+c_{1} C_{2} \kappa_{2}+c_{2} C_{1}^{r} \kappa_{2}\right)}{c_{1}^{2} e_{2}^{2}+\left(c_{1} C_{2}+c_{2} C_{1}^{r}\right)\left(c_{1} \kappa_{2}+c_{2} \kappa_{1}^{r}\right)} \\
& \kappa^{i}=\frac{c_{1}\left\{c_{1}^{2} e_{2}^{4} \kappa_{1}^{i}+\left(c_{1} C_{2}+c_{2} C_{1}^{r}\right)^{2} \kappa_{2}^{2} \kappa_{1}^{i}+e_{2}^{2}\left[2 c_{1}\left(c_{1} C_{2}+c_{2} C_{1}^{r}\right) \kappa_{2} \kappa_{1}^{i}-c_{2}^{2} \kappa_{1}^{r 2} C_{1}^{i}\right]\right\}}{\left[c_{1}^{2} e_{2}^{2}+\left(c_{1} C_{2}+c_{2} C_{1}^{r}\right)\left(c_{1} \kappa_{2}+c_{2} \kappa_{1}^{r}\right)\right]^{2}}
\end{aligned}
$$

We note the following interesting results: i) the nonzero piezoelectric loss moduli $e^{\mathrm{i}}$ is related to the product of the piezoelectric constant $e_{2}$ of the ceramic and loss moduli $C_{1}^{i}$ and $\kappa_{1}^{i}$ of the polymer; ii) As seen in Fig. 1, since $\kappa_{1}^{i}$ is negative and $C_{1}^{i}$ is positive, $e^{i}$ may change sign for different volume fractions (note that the sign convention for dielectric loss moduli here is different from the usual choice); iii) the dielectric loss moduli $\kappa^{\prime}$ and elastic loss moduli $C$ of the piezoelectric composite may exceed the corresponding constants in uncoupled composites due to the piezoelectric coupling.

Turning to more realistic microstructures, we computed the effective complex moduli of both fiberous and laminated two-phase composites by applying the correspondence principle along with the Mori-Tanaka mean-field approach. ${ }^{43-45}$ Complete details are given elsewher ${ }^{42}$ but when the matrix is uncoupled and lossy and the reinforcement (fibers or lamina) is piezoelectric and lossless, closed-form expressions for the effective moduli simplify considerably and these are given in Tables 1 and 2. In the results, the piezoelectric phase is taken to be transversely isotropic with $x_{3}$ as the unique axis. In the composites the $x_{3}$ axis is then aligned with the fibers or normal to the lamina so that the composites are also transversely isotropic. To simplify the presentation of the results, we use the well-known Hill moduli $k=\left(C_{11}+C_{12}\right) / 2$ and $m=\left(C_{11}-C_{12}\right) / 2$, and we use the terminology $\mathrm{X}_{\mathrm{ij} \mid \mathrm{k}}(\mathrm{k}=1,2)$ to denote a tensor quantity $\mathrm{X}_{\mathrm{ij}}$ (in the contracted Voigt notation) associated with phase 1 or 2 .

\section{NUMERICAL RESULTS AND DISCUSSION}

To demonstrate the applicability of the general theory, we present numerical results for a composite consisting of a PZT5A ceramic embedded in an epoxy matrix. The independent material moduli of the two phases used in the calculation are $C_{11}$ $=6.37+\mathrm{i} 0.034 \mathrm{GPa}, C_{12}=3.58-\mathrm{i} 0.012 \mathrm{GPa}, \kappa_{11} / \kappa_{0}=4.431-\mathrm{i} 0.059$ for the isotropic epoxy (where the permittivity of free space, $\kappa_{0}=8.85 \times 10^{-12} \mathrm{C}^{2} \mathrm{Nm}^{2}$ ), and $C_{11}=121 \mathrm{GPa}, C_{12}=C_{13}=75.2 \mathrm{GPa}, C_{33}=111 \mathrm{GPa}, C_{44}=21.1 \mathrm{GPa}, e_{31}=-5.4 \mathrm{C}^{2} / \mathrm{m}$, $e_{33}=15.8 \mathrm{C}^{2} / \mathrm{m}, e_{15}=12.3 \mathrm{C}^{2} / \mathrm{m}, \kappa_{11} / \kappa_{0}=916$, and $\kappa_{33} / \kappa_{0}=830$ for the PZT-5A. In interpreting the results presented here and the more complete results presented elsewhere, ${ }^{42}$ it is worthwhile noting that $\Sigma_{i J} Z_{J i}$ represents the piezoelectric enthalpy rather than the internal energy, and is not necessarily positive. The overall internal energy dissipated by a passive material, which is related to the piezoelectric enthalpy, however, remains positive.

Figure 2 shows the complex piezoelectric moduli $e_{31}, e_{33}$, and $e_{15}$ for a fibrous composite. The results are presented in terms of a map of the real vs. imaginary parts of the constants as the volume fraction of the piezoelectric ceramic increases from zero to unity. Peaks occur in the loss moduli for all three constants because the loss moduli vanish at volume fractions of zero and unity. Between these two limits, the interaction between the piezoelectric effect of the fibers and the elastic and electric losses of the polymer contributes to the nonzero piezoelectric loss moduli of the composite. The imaginary parts of $e_{31}$ and $e_{33}$ are caused by the elastic loss in the polymer, and thus, they are positive. This is because the strain components they are associated with, $\varepsilon_{11}$ and $\varepsilon_{33}$, are uncoupled from the electric field in polymer matrix due to the fibrous 
configuration. Thus the dielectric loss in the polymer has no effect on $e_{31}$ and $e_{33}$ in these particular deformation mode. The imaginary part of $e_{15}$ changes sign as the volume fraction increases. This is because the piezoelectric constant $e_{15}$ is associated with the strain component $\varepsilon_{13}$, which when present induces an electric displacement $D_{1}$ in the piezoelectric ceramic. Since $D_{1}$ is continuous across fiber-matrix interfaces, $D_{1}$, as well as $E_{1}$, will be induced in the polymer matrix. Thus both the elastic and dielectric loss in the polymer contribute to the imaginary part of $e_{15}$. It is also noted that there is a peak in the real part of $e_{33}$. This is because $D_{3}=e_{33} \varepsilon_{33}+e_{11}\left(\varepsilon_{11}+\varepsilon_{22}\right)$, where $e_{33}$ is positive and $e_{11}$ is negative. When $\varepsilon_{33}$ is applied, $\varepsilon_{11}$ and $\varepsilon_{22}$ in the ceramic will have signs opposite of $\varepsilon_{33}$ due to the Poisson effect. Thus the initial addition of polymer (as $c_{2}$ decreases from unity) will enhance the average $D_{3}$ in the composite, and lead to a higher effective $e_{33}$.

Figure 3 shows the complex piezoelectric moduli $e_{31}, e_{33}$, and $e_{15}$ for a laminated composite. The results are again presented in terms of a map of the real vs. imaginary parts of the constants as the volume fraction of the piezoelectric ceramic increases from zero to unity. The imaginary parts of $e_{15}$ is positive because only the elastic losses of the polymer contribute to it. This is because for the laminate configuration, the strain component $\varepsilon_{13}$ is uncoupled from the electric field in the polymer. Both the elastic and dielectric losses contribute to the imaginary parts of $e_{31}$ and $e_{33}$, but they are apparently dominated by the dielectric losses of the polymer.

In order to validate the theory, we fabricated a series of PZT-5A reinforced epoxy laminates with different PZT-5A volume fractions. We used a three-component Marx oscillator $^{46,47}$ to measure the real and imaginary parts of Young's modulus of the epoxy and of the composite samples. We found $E_{11}^{r}=2.7 \mathrm{GPa}$, and $Q^{-1}=E_{11}^{i} / E_{11}^{r}=0.043^{48}$ for the epoxy. Figure 4 shows the measured and predicted loss tangent of the composites $\left(Q^{-1}=E_{11}^{i} / E_{11}^{r}\right)$ as function of volume fraction of PZT-5A. For the composite, the layering is normal to the $x_{3}$ axis and so the $x_{1}-x_{2}$ plane is isotropic. We considered two cases in the calculations. First, we ignored the mechanical and dielectric losses in the ceramic, and only considered these losses in the polymer. As a result, the theoretical prediction underestimated the measured loss tangent of the composite, although it agrees qualitatively. We attributed this discrepancy to the fact that the ceramic also experiences losses. We attempted to account for the mechanical and dielectric losses of PZT-5A using $Q_{M}=75$ and $Q_{E}=50$ as obtained for a resonating thin disc. ${ }^{49}$ This data, though, is not sufficient to fully characterize the transversely isotropic ceramic as, for example, this gives $Q_{M}=\operatorname{Re}\left(C_{11}-C_{13}^{2} / C_{33}\right) / \operatorname{Im}\left(C_{11}-C_{13}^{2} / C_{33}\right)$. As an initial estimate, then, we assumed the loss moduli of PZT-5A are isotropic with $C_{12}^{i}=-C_{11}^{i} / 2$. With these considerations, the theoretical predictions show better agreement with experiment, but now overestimate the loss tangent of the composite. This is probably because we do not accurately know the complete set of complex moduli of the ceramic.

\section{CONCLUSIONS}

We studied the viscoelectroelastic behavior of heterogeneous piezoelectric solids. We coupled the correspondence principle with simple one-dimensional analyses and the Mori-Tanaka micromechanics model for three-dimensional composites to predict the overall behavior of piezoelectric composites comprised of phases with complex moduli. We gave explicit expressions for the effective complex electroelastic moduli of two-phase composites consisting of a lossless piezoelectric phase embedded in a lossy matrix. Numerical results for the complex piezoelectric moduli were presented and discussed, and reasonable agreement was observed between predictions and measurements for laminated composites. We found that the inclusion of losses in the piezoelectric ceramic improved the agreement between predictions and theory.

\section{ACKNOWLEDGEMENTS}

The support of the National Science Foundation (grant number CMS 940-9840) is gratefully acknowledged. We are also grateful to Dr. Hassel Ledbetter and Mr. Sudook Kim of NIST, Boulder for help with the measurements and Dr. Donald Radford and Mr. Fu of Colorado State University for help fabricating the composite specimens. 


\section{REFERENCES}

1. A. S. Nowick and B. S. Berry, Anelastic Relaxation in Crystalline Solids, (Academic Press, New York, 1972).

2. J. D. Ferry, Viscoelastic Properties of Polymers, (Wiley, New York, 1980).

3. R. S. Lakes, Viscoelastic solids, (CRC Press, 1999).

4. J. Meixner and H. G. Reik, Handbuch der Physik III/2, 482 (1959).

5. A. S. Nowick and W. R. Heller, Adv. In Phys. 14, 101 (1965).

6. H. E. Muser and J. Petersson, Fortschritte der Physik 19, 559 (1971).

7. R. Holland, IEEE Trans. Sonics Ultrasonics SU-14, 18 (1967).

8. H. E. Muser and H. Schmidt, Journal de Physique 33, C2, 103 (1972).

9. Arlt and H. Dederichs, Ferroelectrics 29, 47 (1980).

10. Arlt, Ferroelectrics 40, 149 (1982).

11. J. G. Smits, IEEE Trans. Sonics Ultrasonics SU-23, 393 (1976).

12. T. Furukawa, J. Aiba, and E. Fukada, J. Appl. Phys. 50, 3615 (1979).

13. J. Bur, J. Biomechanics 9, 495 (1976).

14. T. Furukawa and E. Fukada, Jap. J. Appl. Phys. 16, 453 (1977).

15. S. Sherrit, H. D. Wiederick, B. K. Mukherjee, M. and Sayer, Ferroelectrics 193, 89 (1997).

16. T. Furukawa, K. Fujino, and E. Fukada, Jap. J. Appl. Phys. 15, 2119 (1976).

17. G. S. Kumar and G. Prasad, J. Mater. Sci. 28, 2545 (1993).

18. R. E. Newnham, D. P. Skinner, and L. E. Cross, Mater. Res. Bull. 13, 525 (1978).

19. A. A. Grekov, S. O. Kramarov, and A. A. Kuprienko, Ferroelectrics 99, 115 (1989).

20. H. L. W. Chan and J. Unsworth., IEEE Trans. Utrasonics Ferroelect. Freq. Contr. 36, 434 (1989).

21. H. Cao, Q. M. Zhang, and L. E. Cross, J. Appl. Phys. 72, 5814 (1992).

22. M. L. Dunn and M. Taya, Proc. R. Soc. Lond. A 443, 265 (1993).

23. M. L. Dunn and M. Taya, Int. J. Solids Struct. 30, 161 (1993).

24. T. Chen, 1994b, Int. J. Solids Struct. 31, 3099 (1994).

25. T. Chen, Composites, 27B, 467 (1996).

26. J. Y. Li and M. L. Dunn, J. of Intelligent Material Systems and Struct. 9, 404 (1998)

27. Y. Benveniste. and G. J. Dvorak, J. Mech. Phys. Solids 40, 1295 (1992).

28. T. Chen, J. Mech. Phys. Solids 41, 1781 (1993).

29. Y. Benveniste, J. Appl. Mech. 60, 265 (1993).

30. Y. Benveniste, J. Appl. Mech. 60, 270 (1993).

31. Y. Benveniste, Proc. R. Soc. Lond. A 441, 59 (1993).

32. M. L. Dunn, Proc. R. Soc. Lond. A 441, 549 (1993).

33. J. Y. Li and M. L. Dunn, Int. J. Eng. Sci. 37, 665 (1999).

34. T. Olson and M. Avellaneda, J. Appl. Phys. 71, 4455 (1992).

35. P. Bisegna and R. Luciano, J. Mech. Phys. Solids, 44, 583 (1996).

36. M. Hori and S. Nemat-Nasser, Mechanics of Materials 30, 1 (1998).

37. J. Li and G. J. Weng, Composites Engineering 4, 1073 (1994).

38. Z. Hashin, J. Appl. Mech. 32, 330 (1965).

39. Z. Hashin, ALAA J. 4, 1411 (1966).

40. M. L. Dunn, J. Acoust. Soc. Amer. 98, 3360 (1995).

41. D. M. Barnett and J. Lothe, Phys. Status Solidi (b) 67, pp. 105 (1975).

42. J. Y. Li and M. L. Dunn, in preparation.

43. T. Mori and K. Tanaka, Acta Mettall. 21, 571 (1973).

44. T. Mura, Micromechanics of Defects in Solids, second ed. (Martinus Nijhoff, Dordrecht, The Netherlands, 1987).

45. S. Nemat-Nasser and M. Hori, Micromechanics: Overall Properties of Hetero-geneous Materials, (Elsevier, NorthHolland, 1993).

46. J. Marx, J., The Review of Scientific Instruments 22, 503 (1951).

47. H. M. Ledbetter, Cryogenics, November, 637 (1980).

48. H. M. Ledbetter, private communication.

49. TP-226, Important Properties of Morgan Matrox Piezoelectric Ceramics, Morgan Matrox, Inc. 


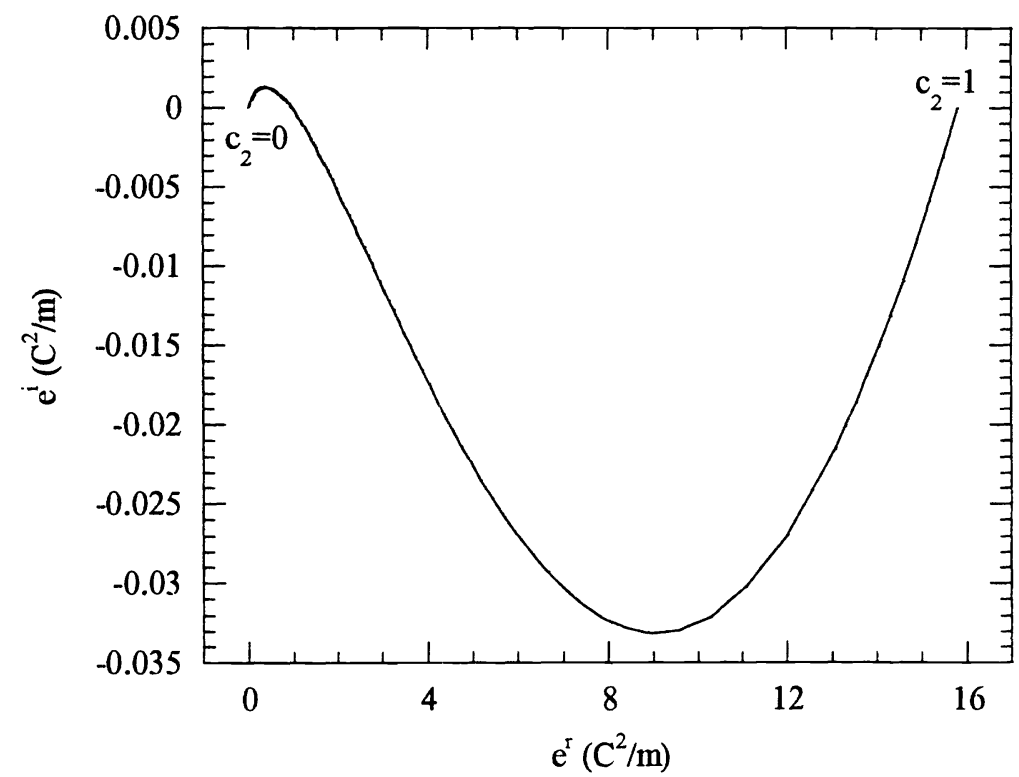

Fig. 1 Real-Imaginary component map of the piezoelectric modulus of a one-dimensional composite where the phases are arranged in series. In the calculation, we have used $C_{1}^{c}=6.37+0.157 i \mathrm{GPa}$, $\kappa_{1}^{c} / \kappa_{0}=4.431-0.0585 i, C_{2}=111 \mathrm{GPa}, \kappa_{2} / \kappa_{0}=830$, and $e_{2}=15.8 \mathrm{C}^{2} / \mathrm{m}$.

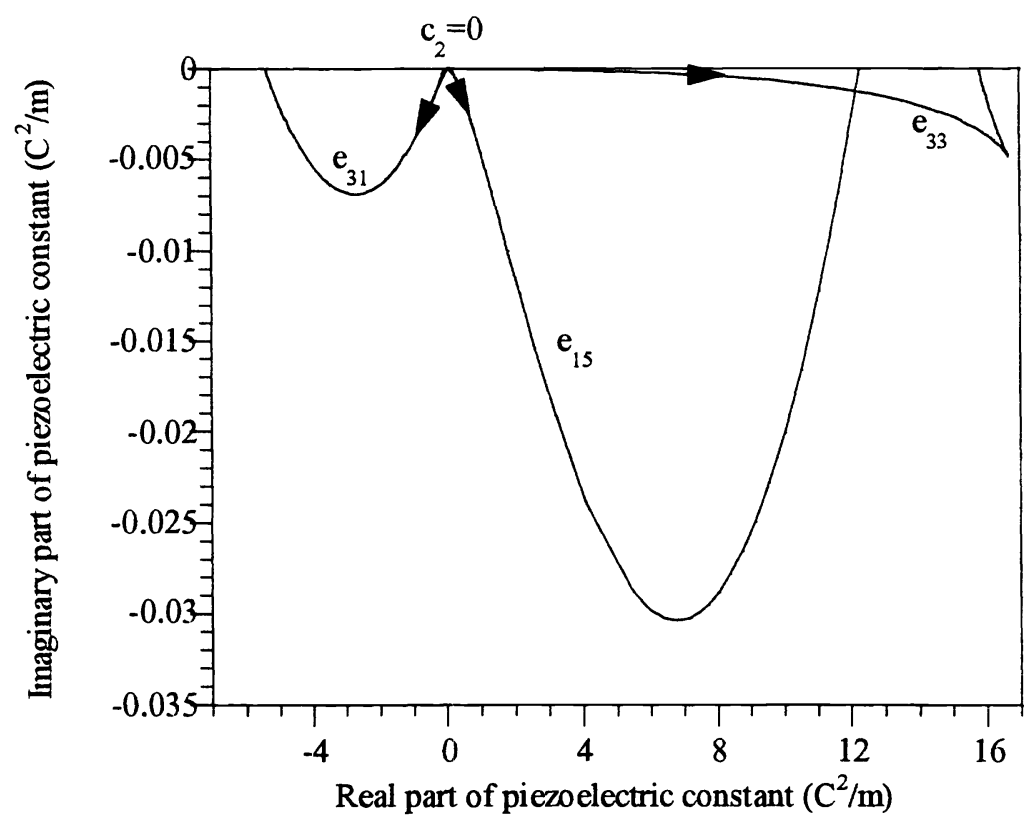

Fig. 2 Real-Imaginary component map of the piezoelectric moduli of a fibrous composite. The arrows denote the direction along the curve of increasing volume fraction, $c_{2}$. 


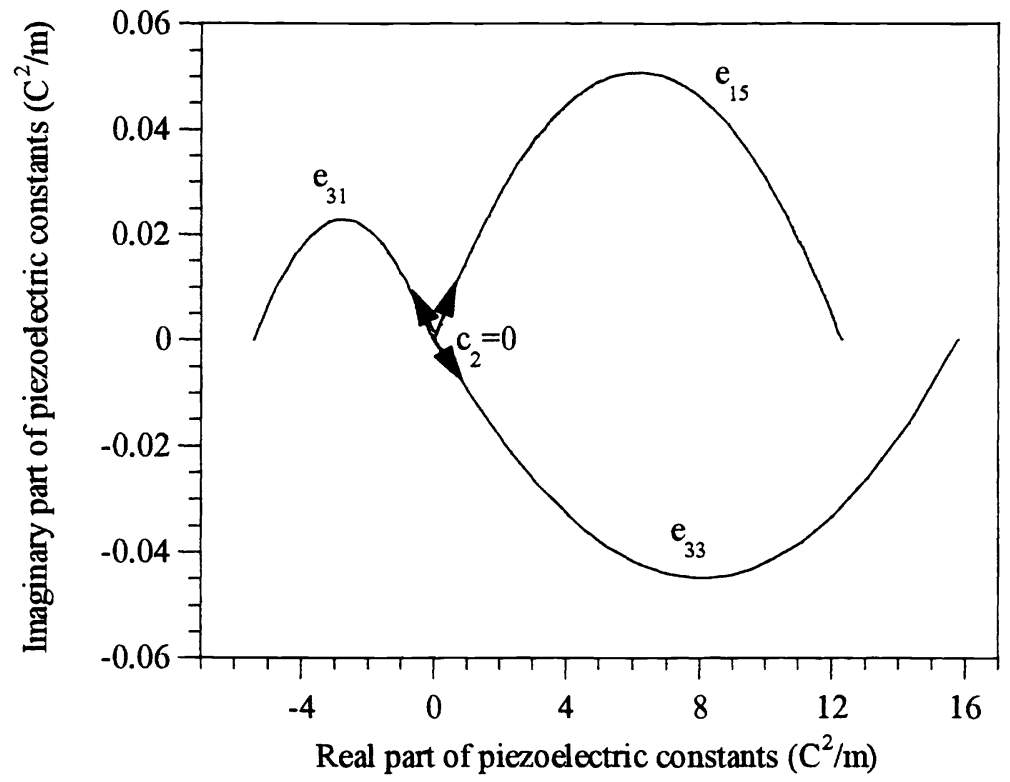

Fig. 3 Real-imaginary component map of the piezoelectric moduli of a fibrous composite. The arrows denote the direction along the curve of increasing volume fraction, $c_{2}$.

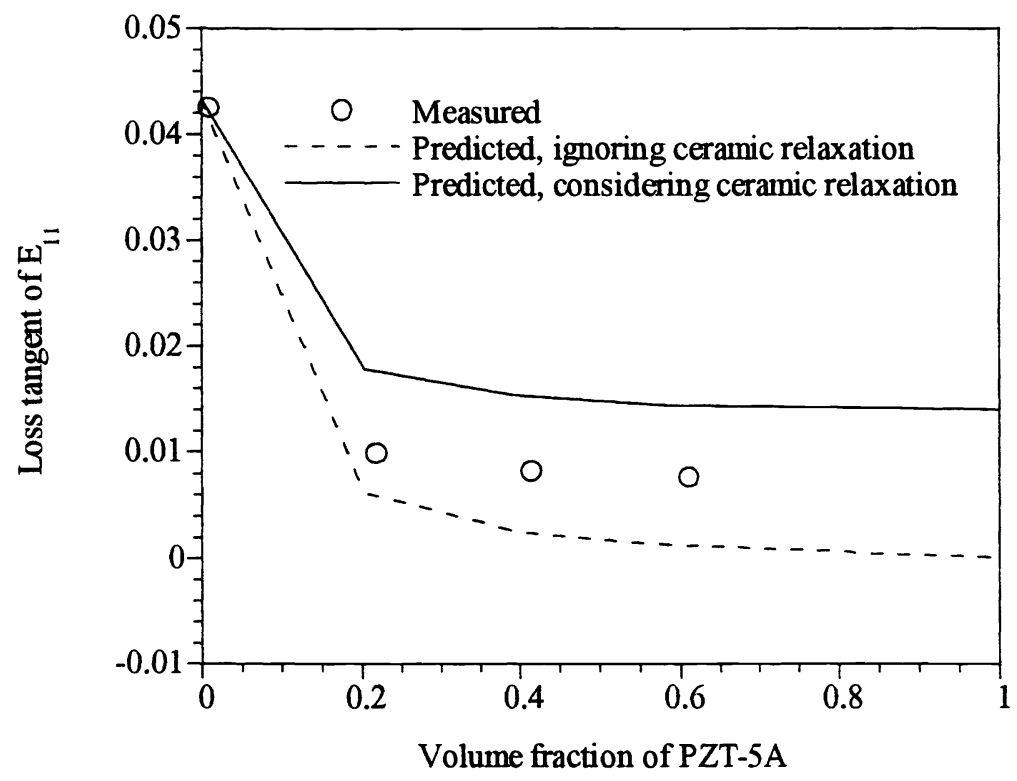

Fig. 4 Loss tangent of Young's modulus $E_{11}$ of PZT-5A reinforced epoxy laminate. 







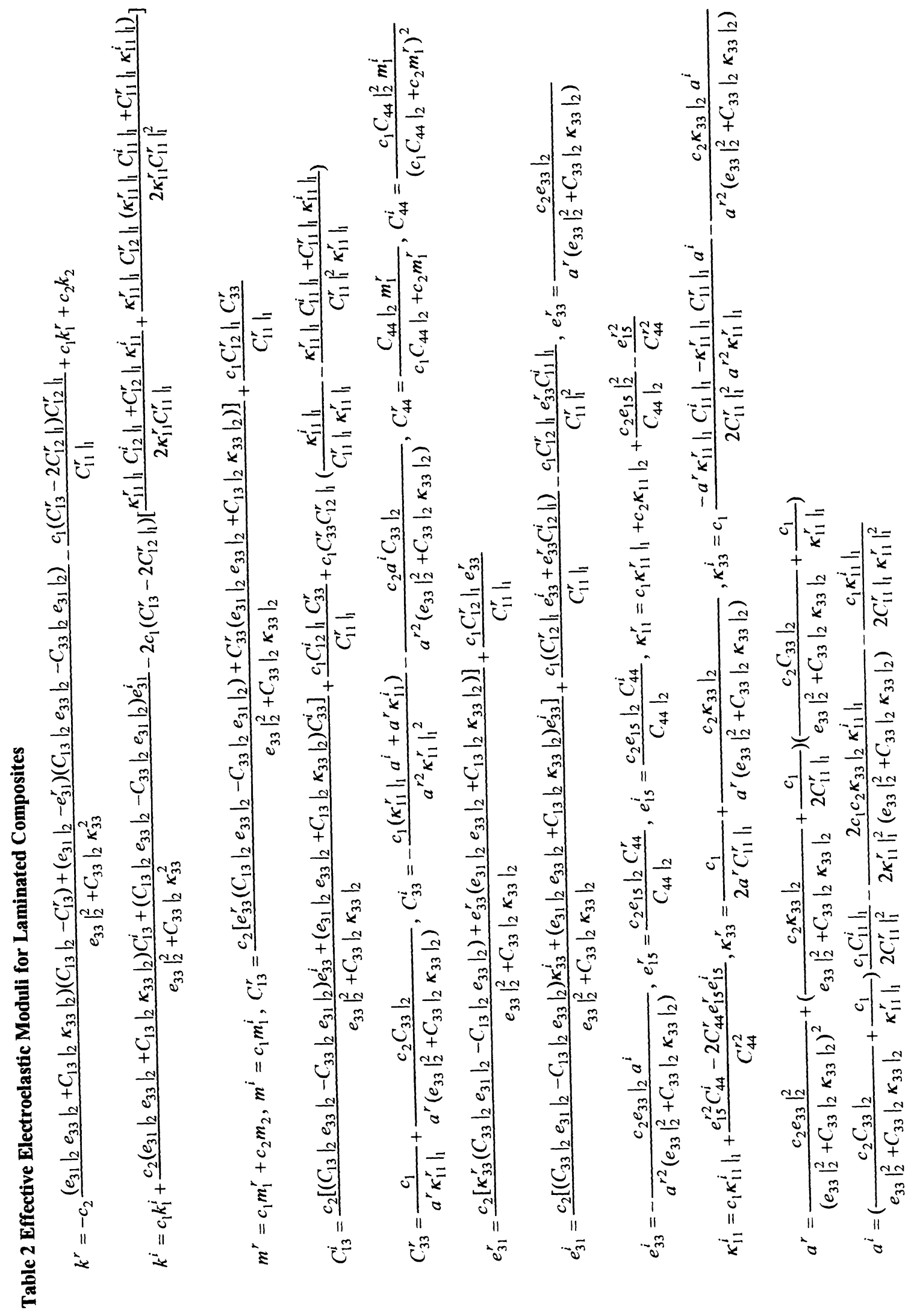

